\title{
Towards Wearable Capacitive Sensing of Physiological Parameters
}

\author{
Jingyuan Cheng \\ Embedded Systems Lab \\ University of Passau \\ Passau, Germany \\ jingyuan.cheng@uni-passau.de
}

\author{
Paul Lukowicz \\ Embedded Systems Lab \\ University of Passau \\ Passau, Germany
}

\begin{abstract}
We describe an initial investigation of the suitability of body worn capacitive sensors for the measurement of physiological parameters. We describe the basic principle, our measurement setup, and initial signal examples obtained using a conductive textile electrode. The examples show that breathing, breathing depth, and even drinking a cup of water produce distinguishable signals.
\end{abstract}

Keywords: wearable sensing, textile sensors, physiological sensing

\section{INTRODUCTION}

The ability to measure physiological parameters with unobtrusive, wearable sensors is an important feature of many pervasive health care systems. In this paper we describe initial results of using wearable capacitive sensors for this purpose. Capacitive sensing is widely used in industrial applications ranging from distance sensors, through intrusion detection, to quality inspection. Simply said the appeal of capacitive sensing stems from the fact, that allows us to analyze the structural and material properties inside closed spaces. For example, in industrial applications, capacitive sensors are used to check the content of sealed packages on a conveyor belt.

\section{A. Contributions with Respect to Related Work}

Some initial work on using capacitive sensing in pervasive computing was done at MIT where such sensors were used as distance sensors to measure limbs angles in a dance monitoring system ${ }^{[1]}$. The Applied Electronics Laboratory in Helsinki University of Technology has developed a set of smart carpet to trace footsteps and to measure breath and heartbeat when a person falls onto the ground ${ }^{[2]}$. A system for step recognition has also been developed by Future Shape $\mathrm{GmbH}^{[3]}$. Rob MacLachlan from CMU has demonstrated a capacitive system able to detect a person breathing from a distance of $30 \mathrm{~cm}$ away from the chest.$^{[4]}$

Outside the pervasive computing area there have been considerable interests in the use of capacitive sensing for complex 3D imaging ${ }^{[5]}$.

The main contribution of this work is to demonstrate that relevant physiological signals can be acquired with a conductive textiles based, wearable setup connected to simple low power electronics. Specifically we show that the breathing rate, the depth of breath, and even drinking can produce distinguishable signals.

Permission to make digital or hard copies of all or part of this work for personal or classroom use is granted without fee provided that copies are not made or distributed for profit or commercial advantage and that copies bear this notice and the full citation on the first page. To copy otherwise, to republish, to post on servers or to redistribute to lists, requires prior specific permission and/or a fee.

PERVASIVEHEALTH 2008, 30 Jan - 1 Feb. Tampere, Finland

Copyright $@ 2008$ ICST 978-963-9799-15-8

DOI 10.4108/ICST.PERVASIVEHEALTH2008.2517
We describe our system, the experimental setup and discuss issues in ongoing system improvement.

\section{Basic PRinciple And Measurement SetuP}

\section{A. Measurement Principle}

An ideal capacitor is made of two conductive plates separated and insulated from each other by a dielectric. Its capacitance $(\mathrm{C})$ is defined as the ratio of $\operatorname{charge}(\mathrm{Q})$ to voltage $(\mathrm{V})$. The value depends on the plates' area, distance between, and the dielectric's material.

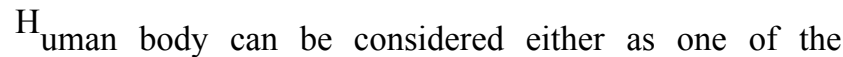
electrodes when connected directly to the wire or as the dielectric when separated from the other electrode by another thin dielectric with higher resistance. In the latter case, the capacitance of the system is determined by the dielectric properties of the inside of the human body. Thus body's change in shape (including changes of shapes of internal organs) as well as substances (air when breathing, water when drinking) can cause a detectable change in capacitance.

\section{B. Measurement Setup}

Our measurement method is shown in Fig. 1. A processing unit controls the voltage at point a, setting it to logic ' 1 ' (3.0V) 睡 charge the capacitor $\mathrm{C}$ through $\mathrm{R}$, or logic ' 0 ' $(0 \mathrm{~V})$ to discharge. The voltage at $\mathrm{b}$ is then compared with the voltage

$\mathrm{c}$, which is set to either $0.825 \mathrm{~V}$ or $1.65 \mathrm{~V}$ by the processing unit. Thus the output $\mathrm{d}$ indicates whether the voltage at $\mathrm{c}$ is greater $(d=0)$ or less $(d=1)$ than the voltage at $b$. Because the time needed for voltage to raise from $0.825 \mathrm{~V}$ to $1.65 \mathrm{~V}$ (e.g. t1) or fall from $1.65 \mathrm{~V}$ to $0.825 \mathrm{~V}$ (e.g. t2) is proportional to the capacitance, variation of $\mathrm{C}$ can be achieved by measuring the time required for charging or discharging the capacitor.

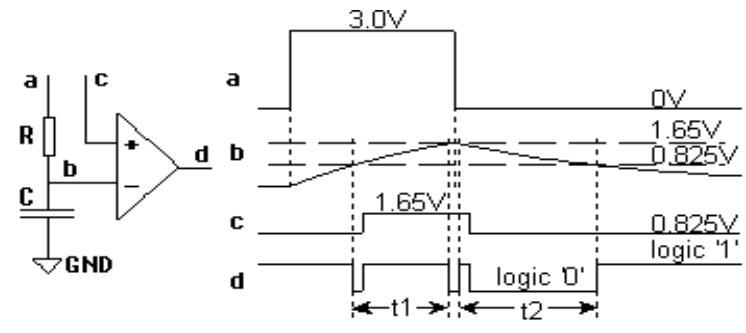

Fig.1. Measuring principle and voltage at points a,b,c,d 


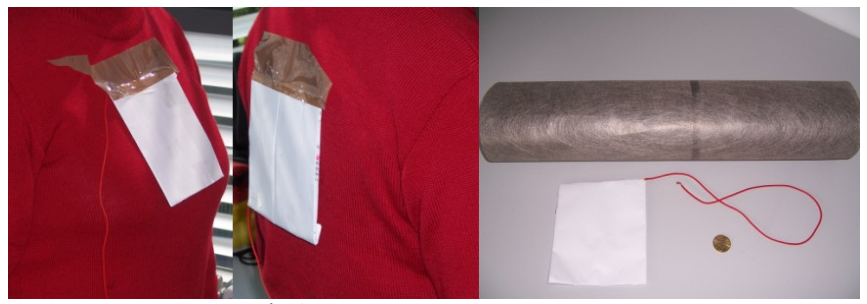

Fig.2. Measurement setup

The experimental setup is shown in Fig. 2, with conductive textile as electrode of the capacitor. To achieve adequate measurement precision, $\mathrm{R}$ should be no less than $1 \mathrm{M} \Omega$ (the resistance of a person is much smaller than that). Hence the conductive textile is wrapped up in paper to avoid touching the person's skin directly. Figure 2 illustrates the placement of the electrodes. For the actual measurement the electrodes were inserted between two layers of clothes. The comparator and the control signals are provided by multi-channels MSP msp430f2131. For better time resolution, an external clock is provided to the MSP. Data is sent to the PC via serial port.

\section{INITIAL RESULTS}

\section{A. Breath}

Fig. 3 shows the result of breath measurement. The electrode is a conductive textile with an area of $10 \times 10 \mathrm{~cm}^{2}$ put on the chest. The horizontal axis is the measurement time in second. The vertical shows the time it takes to charge the capacitor in 'clock ticks' of the MSP (which, as already explained, is proportional to the capacitance). The difference between normal breathing (100s 210s) and deep breath $(210 \mathrm{~s} \sim 360 \mathrm{~s}, 360 \mathrm{~s} \sim 500 \mathrm{~s})$ is very clear. By searching for peaks in frequency domain, the frequency of breathing can also be determined, as shown in Fig. 4. The vertical value is related to the volume of air (the exact correlation between signal and air volume has not been studied in detail yet).

\section{B. Drinking Water}

Clearly drinking causes a change to body capacitance, especially if the electrode is located in the stomach region.

Fig. 5 shows respective experimental results obtained with our system (green for electrode on the breast and red for electrode on the back). From $50 \mathrm{~s}$ to $70 \mathrm{~s}$ the person drinks about $150 \mathrm{ml}$ of water.

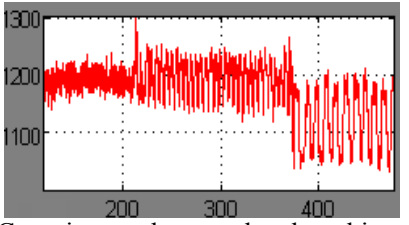

Fig.3. Capacitance change when breathing

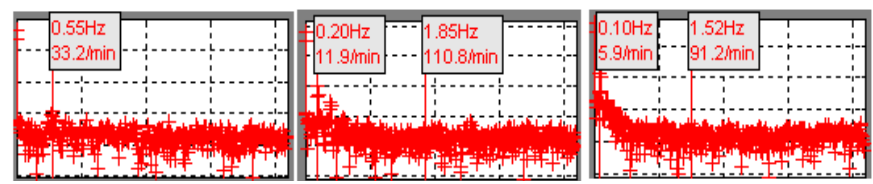

Fig.4. Frequency domain analyse(100s $\sim 210 \mathrm{~s} / 210 \mathrm{~s} \sim 360 \mathrm{~s} / 360 \mathrm{~s} \sim 500 \mathrm{~s})$

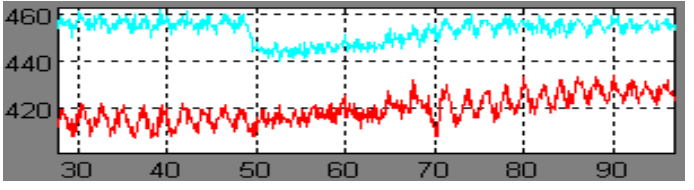

Fig.5. Capacitance change when drinking water

It can be seen that while drinking, the capacitance on front electrode changes to a quite low level. This is because the person has to raise the hand with the bottle of water near the electrode before drinking. After the bottle is put back, the capacitance rises back again. However, there remains a distinguishable difference between before and after drinking. The capacitance on the back doesn't change much during drinking, because the bottle is far away from it, and the variation during drinking period is also comparatively small because human tend to stop breathing or slow down breathing speed while drinking. After drinking, the capacitance rises. Meanwhile breath frequency can still be detected.

\section{CONCLUSION AND FUTURE WORK}

We have demonstrated that a unobtrusive, textile electrode and a simple, low power circuit can detect capacitance change related to breathing and drinking a cup of water. Clearly this work is preeliminary. The current setup has no outside shielding and is very sensitive to disturbances as objects (or hands) are moved around it. Thus outside static lab environments the signals tend to be buried in noise. We will address this problem by using shielding and a more advance measuring chip (Freescale MC34940 featuring shield for cable and electrode ${ }^{[6]}$. The new chip should also provide more sensitivity. We will also investigate different signal processing algorithms to reduce noise and improve signal quality. This might enable us to measure further parameters such as heart rate. Finally statistically relevant, systematic experiments need to be performed to evaluate system accuracy under different conditions.

\section{REFERENCES}

[1] Ryan Aylward, Joseph A. Paradiso, "Sensemble: a wireless, compact, multi-user sensor system for interactive dance", Proceedings of the 2006 International Conference on New Interfaces for Musical Expression

[2] H.Rimminen, L.Westling, and A.Paukkunen, "A novel method for monitoring persons in indoor environment", ISBN-13 978-951-22-85479,ISBN-10 951-22-8547-9

[3]Christl Lauterbach (invited), SensFloor®: A large-area Sensor System for Ambient Assisted Living, Smart Fabrics 2007 Conference, Washington, 09.05.2007

[4]Rob MacLachlan,Spread Spectrum Capacitive Sensor, http://www.cs.cmu.edu/ ram/capsense/index.html, personal webpage

[5] Warsito Warsito, Qussai Marashdeh, Liang-Shih Fan, "Electrical Capacitance Volume Tomography",IEEE Sensors Journal, Vol.7 No.4, Apr.2007, 525-535

[6] Freescal semiconductor, "Electric Field Imaging Device, document number MC34940", datasheet 